\title{
Application of Occlusal Splint for Conservative Management of Pediatric Mandibular Body Fractures
}

\author{
Akifumi Enomoto, PhD and DDS ${ }^{1 *}$, Kazuhide Matsunaga ${ }^{1}$, Takao Mukai, PhD and DDS ${ }^{1}$, \\ Takeshi Shimoide PhD and DDS ${ }^{2}$, Suguru Hamada PhD and DDS $^{3}$ \\ ${ }^{1}$ Associate professor, Department of Oral and Maxillofacial Surgery, Kindai University, Faculty of Medicine. \\ ${ }^{2}$ Assistant professor, Department of Oral and Maxillofacial Surgery, Kindai University, Faculty of Medicine. \\ ${ }^{3}$ Professor, Department of Oral and Maxillofacial Surgery, Kindai University, Faculty of Medicine. \\ *Corresponding Author: Akifumi Enomoto, PhD and DDS, Department of Oral and Maxillofacial \\ Surgery, Kindai University School of Medicine, 377-2, Ohno-higashi, Osaka-Sayama, 589-8511, Japan, \\ Email: enomotoa@med.kindai.ac.jp
}

\begin{abstract}
An 8-year-old Japanese boy presented with deranged malocclusion, occlusal contact on the left side only, and displaced vertical fractures with minimal step deformity in the distal region of the left mandibular canine and right mandibular first molar region. As he had mixed dentition, a conservative treatment plan was adopted. We fabricated a maxillary splint comprising autopolymerizing acrylic resin and pivot splints to raise the position of the occlusion on the left side. Three weeks after treatment, panoramic radiography showed that the vertically displaced mandibular segment was repositioned. Two years after treatment, the left mandibular canine had erupted and root development of the right first molar region, where the displaced vertical fractures had existed, was normal. Conservative treatment using occlusal splints is an effective alternative to surgery for multiple mandibular fractures with malocclusion without large buccal-lingual displacement.
\end{abstract}

Keywords: Occlusal splint, pediatric mandibular body fracture, conservative treatment, ongoing mandibular growth

The management of pediatric mandibular treatment is challenging for maxillofacial surgeons due to ongoing mandibular growth involving tooth buds [1]. The treatment of such fractures has been a longstanding topic of research. Successful treatment of mandibular fractures signifies an anatomic bony union with restoration of the occlusion.

A mandibular fracture may be either treated conservatively or with open reduction and internal maxillomandibular fixation. When multiple mandibular fractures occur in a child, open reduction and internal maxillomandibular fixation could be often indicated [2, 3]. However, there are concerns about mandibular growth and dentition development with surgical treatment. Therefore, an alternative approach for pediatric patients with mandibular body fracture and malocclusion is conservative treatment using splints with circummandibular wires and intermaxillary fixation. However, treatment by circummandibular fixation with wires and acrylic splints requires general anesthesia or additional sedation for the patient.
We herein present a clinical case in which conservative treatment of pediatric mandible body fractures was achieved with the use of an occlusal splint.

\section{Case Report}

An 8-year-old Japanese boy was referred to our clinic complaining of pain in the right mandibular region. The patient fell while playing the day prior to this visit and hit his cheek on the ground. His medical history was otherwise unremarkable. Intraoral findings showed deranged malocclusion. Occlusal contact was observed on the left side but not the right side (Fig. 1). Panoramic radiography revealed displaced vertical fractures with minimal step deformity located in the distal region of the left mandibular canine and right first molar region in the mandible body (Fig. 2A). Computed tomography examination showed no other fracture in the bony structure other than the mandible. Finally, the diagnosis of mandibular body fracture in the region of the left canine and right first molar was established. 


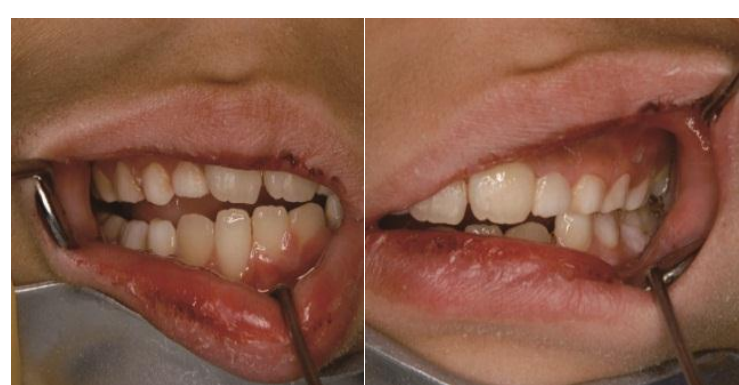

Figure1. Intraoral findings of the occlusal contact. Occlusal contact is observed on the left side but not on the right side
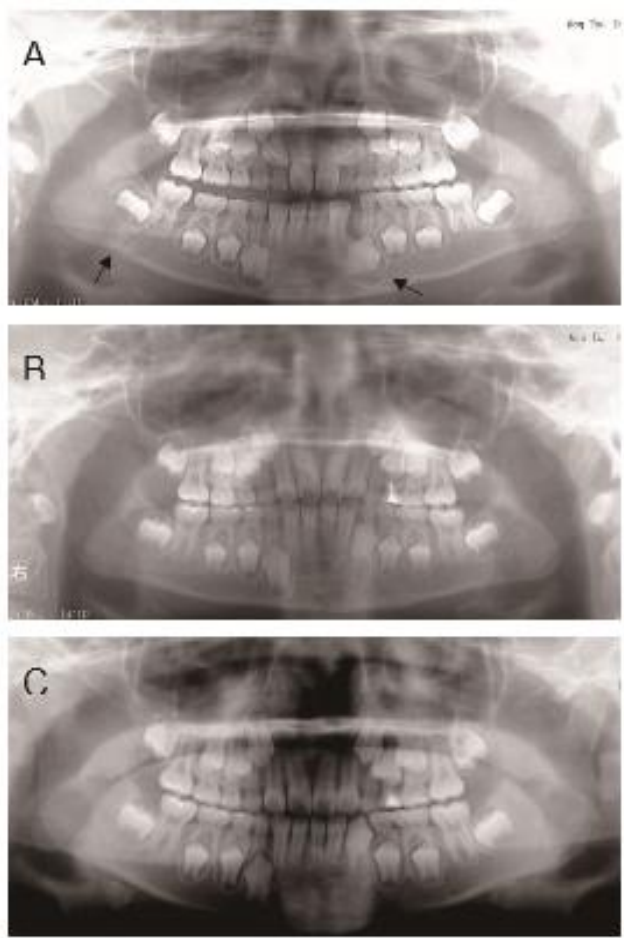

Figure2.

A. Panoramic radiograph at initial presentation shows multiple mandibular fractures located in the distal region of the left mandibular canine and right first molar region in the mandible body. Occlusal contact is observed on the left side but not the right side. Arrows indicate fracture points in the mandible.

B. Panoramic radiograph taken 3 weeks after treatment shows the repositioned mandible where occlusal contact was acquired.

C. Panoramic radiograph taken 2 years after treatment shows the repositioned mandible. The left mandibular canine erupted and root development of the right first molar region was normal. Adverse outcomes were not observed.

The patient had multiple mandibular fractures with malocclusion. However, the two fractures were on the immature permanent teeth of the right first molar and developing bud of the left canine, and the buccal-lingual displacement of the two displaced vertical fractures was not prominent. As the patient had mixed dentition, a conservative treatment plan was preferred and designated.

The patient received a maxillary splint made of autopolymerizing acrylic resin. Pivot-type splints were fabricated to raise the position of the occlusion on the left side and intraorally adjusted (Fig. 3).

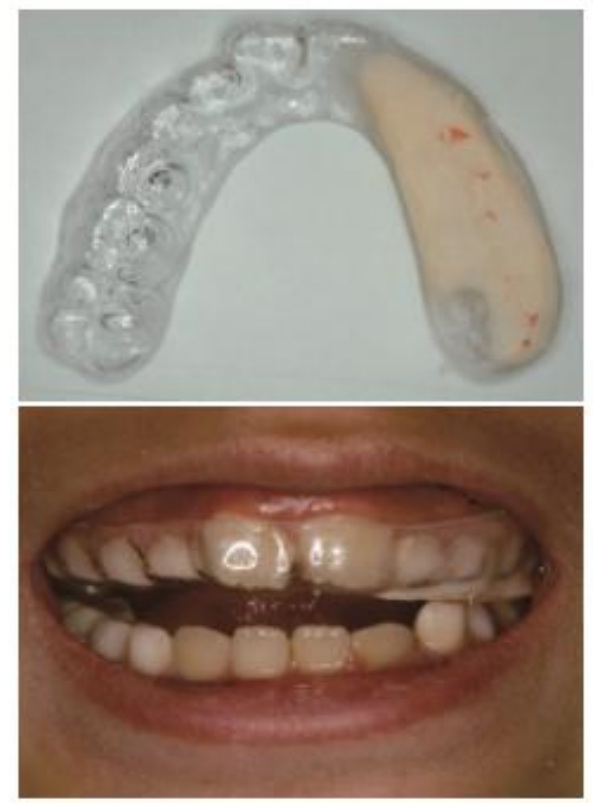

Figure3. The occlusal splint. The split increased the vertical dimension. By the principle of leverage, the right mandibular fracture segment is repositioned.

Conservative movement of the anterior segment of the mandible by occlusal force was induced and repositioned. Finally, occlusal contact was obtained on the right side, and the occlusal state was clinically repositioned by checking with a bite checker 3 weeks after treatment. Panoramic radiography showed that the vertically displaced mandibular segment was repositioned (Fig. 2B).

At the 2-year follow-up, the left mandibular canine was erupted and root development of the right first molar region, where the displaced vertical fractures had existed, was normal (Fig. $2 \mathrm{C})$.

\section{DISCUSSION}

This report showed the successful recovery of pediatric multiple mandible fractures by using conservative treatment with the occlusal splint.

About $40 \%$ of pediatric patients with maxillofacial fractions have mandibular fractures, around half of whom have multiple mandibular fractures [2-4]. The most common multiple mandibular fracture pattern is angle and 
contralateral parasymphysis [3]. The present case also exhibited this pattern. Generally, surgical treatment is performed for multiple fractures with malocclusion. When surgical operation is performed, the management should be minimally invasive with respect to future mandibular growth, development, and function. Titanium miniplates and screws or the biodegradable fixation system can be used [5]. However, operatively managed fractures are significantly more likely to result in adverse outcomes than conservatively managed fractures [3]. Additionally, medical costs for surgical treatment are much higher than that of conservative management.

Even if splints with circummandibular wires and intermaxillary fixation were conservatively adopted for a mandibular fracture with malocclusion, general anesthesia or intravenous sedation would be necessary, even without surgical operation.

The present case showed multiple mandibular fractures and malocclusion. The buccal-lingual displacement of two displaced vertical fractures in the present case was not prominent. The two fractures were on immature permanent teeth of the right first molar and developing bud of the left canine. Therefore, conservative treatment was adopted. Conservative gradual repositioning was expected by using an occlusal splint. The occlusal splint increased the occlusal vertical dimension, subsequently increasing the occlusal bite force. The contact point of the splint and left mandible molar works as a fulcrum, the right masseter muscle works as a point of application, and the fracture segment of the right mandible works as the action point of a lever. Therefore, the right mandibular fracture segment could be repositioned [6].

The occlusal splint was removed and repositioning of the mandibular segment and bite position could be observed during the treatment period. While the mandibular segment was repositioned, the bite position could be visually inspected by using bite-check paper. Additionally, general anesthesia or intravenous sedation was not necessary for this treatment. Thus, the present treatment avoided adverse outcomes associated with surgical treatment and was more cost-effective. Even if the patient exhibits multiple mandibular fractures and malocclusion, conservative treatment using an occlusal splint could offer an effective treatment alternative to surgery.

\section{CONClusion}

Conservative treatment using an occlusal splint could offer an effective treatment alternative to surgery, even if the patient exhibits multiple mandibular fractures and malocclusion.

\section{REFERENCES}

[1] Aizenbud D, Hazan-Molina H, Emodi O, Rachmiel A, The management of mandibular body fractures in young children, Dent Traumatol. 25, 565-570, (2009).

[2] Glazer M, Joshua BZ, Woldenberg Y, Bodner L, Mandibular fractures in children: analysis of 61 cases and review of the literature, Int J Pediatr Otorhinolaryngol. 75, 62-64, (2011).

[3] Smith DM, Bykowski MR, Cray JJ, et al., 215 mandible fractures in 120 children: demographics, treatment, outcomes, and early growth data, Plast Reconstr Surg. 131, 13481358, (2013).

[4] Al Shetawi AH, Lim CA, Singh YK, Portnof JE, Blumberg SM, Pediatric Maxillofacial Trauma: A Review of 156 Patients, J Oral Maxillofac Surg. 74, 1420 e1421-1424, (2016).

[5] Singh M, Singh RK, Passi D, Aggarwal M, Kaur $\mathrm{G}$, Management of pediatric mandibular fractures using bioresorbable plating system Efficacy, stability, and clinical outcomes: Our experiences and literature review, J Oral Biol Craniofac Res. 6, 101-106, (2016).

[6] Mansour RM, Reynik RJ, In vivo occlusal forces and moments: I. Forces measured in terminal hinge position and associated moments, J Dent Res. 54, 114-120, (1975).

Citation: Akifumi Enomoto, Kazuhide Matsunaga, Takao Mukai,Takeshi Shimoide, Suguru Hamada. Application of Occlusal Splint for Conservative Management of Pediatric Mandibular Body Fractures. ARC Journal of Clinical Case Reports. 2018; 4(1): 13-15. doi:dx.doi.org/ 10.20431/2455-9806.0401005.

Copyright: (C) 2018 Authors. This is an open-access article distributed under the terms of the Creative Commons Attribution License, which permits unrestricted use, distribution, and reproduction in any medium, provided the original author and source are credited. 